\title{
SEMIGROUP OF ENDOMORPHISMS OF A LOCALLY COMPACT GROUP $\left.{ }^{1}\right)$
}

\author{
BY \\ MORIKUNI GOTO AND NAOKI KIMURA
}

Introduction. A homomorphism of a group ( $B$ into itself is called an endomorphism of $(3)$. The set of all endomorphisms of $(\$)$ forms a semigroup $\left({ }^{2}\right)$ containing all automorphisms of $(B)$. If $(5)$ has a topology in which it is a topological group, then it will be natural to limit ourselves to the study of the set of

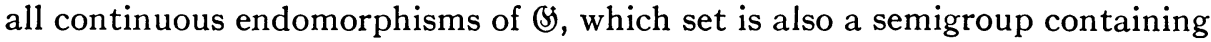
the group of all bicontinuous automorphisms of $\mathbf{B}$. In the present paper we are interested in these semigroups for locally compact topological groups.

Let $G$ be a locally compact group, and let $\widetilde{F}(G)$ be the semigroup of all continuous endomorphisms of $G$. The first step of our investigation is to topologize $\widetilde{\mathfrak{F}}(G)$ in such a way that $\widetilde{\mathfrak{F}}(G)$ becomes a topological semigroup, $\left.{ }^{3}\right)$ which will be done in $\S 1$. The results in $\$ 1$ and in $\$ 2$ will indicate that the topology introduced here is the unique natural one.

$\$ 2$ will be devoted to the special case where $G$ is a Lie group, mainly for the purpose of later use. The fact that any continuous endomorphism of a Lie group induces an endomorphism of the Lie algebra (infinitesimal group) is useful to us.

In $\S 3$ we shall consider the case of compact groups. Let us denote by $\mathbb{E}(G)$ the connected component containing the identity of $\widetilde{F}(G)$. Then in the case in which $G$ is a compact group, $\mathbb{E}(G)$ is composed of bicontinuous automorphisms (Theorem 1), and the structure of the group $\mathbb{E}(G)$ will be determined completely.

In $\S 4$ we consider the group $\tilde{\mathfrak{U}}(G)$ of all bicontinuous automorphisms of a locally compact group $G . \widetilde{\mathscr{A}}(G)$ will be topologized relatively as a subspace of $\widetilde{\mathfrak{F}}(G)$. Then $\mathfrak{\mathfrak { A }}(G)$ is, of course, a topological semigroup. Moreover, under a certain additional condition, we may prove the continuity of the operation of taking inverses in $\tilde{\mathfrak{A}}(G)$ so that $\widetilde{\mathfrak{A}}(G)$ will also be a topological group (Theorem 2). This proof uses certain properties of endomorphisms.

In $\$ 5$ we shall discuss in detail the structure of $\&(G)$ for a locally compact connected group $G$ under the structure theorem of locally compact groups. Most of the theorems and arguments used here are quite analogous to those

Received by the editors September 27, 1956.

(1) This work was done under the support of the National Science Foundation.

(2) A set with a binary operation ( multiplication) is called a semigroup if the operation is associative.

(3) A semigroup is called a topological semigroup if it is a topological space and if multiplication is continuous with respect to both variables. 
in a paper by one of the authors on the automorphism group of $G$. The main results are stated in Theorem 3, Theorem 4 and Theorem 5 .

1. Topology of the semigroup of endomorphisms. Let $G$ be a locally compact topological group $\left({ }^{4}\right)$. By an endomorphism of $G$ we mean a continuous homomorphism from $G$ into itself. Let $\sigma$ and $\tau$ be endomorphisms of $G$. Then the product $\sigma \tau$ defined by $(\sigma \tau)(x)=\sigma(\tau(x)), x \in G$, is also an endomorphism. So the set $\widetilde{E}(G)$ of all endomorphisms of $G$ forms a semigroup. Let now $\tilde{\mathfrak{A}}(G)$ be the subset of $\mathbb{E}(G)$ composed of all bicontinuous automorphisms $\left({ }^{5}\right)$ of $G$. Then $\tilde{\mathfrak{A}}(G)$ clearly forms a group.

In $\$ 1$ we shall consider a natural topology of $\widetilde{\xi}(G)$. Although most of the results here seem to be known, we shall discuss the basic properties of the topology of $\widetilde{E}(G)$ for the sake of completeness.

Let $G$ be a locally compact group. By a nucleus we mean here an open neighborhood $U$ of the identity $e$ whose closure is compact and satisfies $U=U^{-1}$. As is known, all the nuclei form a base of neighborhoods of $e$. For a given triple of an endomorphism $\rho$, a compact subset $\Omega$, and a nucleus $U$ of $G$, we define a subset $(\rho ; \Omega, U)$ of $\widetilde{\&}(G)$ in the following way:

$$
(\rho ; \Omega, U)=\left\{\sigma \mid \sigma \in \widetilde{\mathscr{F}}(G), \rho(x)^{-1} \sigma(x) \in U \text { for all } x \in \Omega\right\}\left(^{6}\right) .
$$

Proposition 1. By considering the set of all possible ( $\rho$; $\Omega, U$ )'s as a base of neighborhoods of $\rho, \widetilde{\mathfrak{F}}(G)$ becomes a topological space satisfying the separation axiom of Hausdorff. Moreover the multiplication of $\widetilde{F}(G): \widetilde{F}(G) \times \widetilde{F}(G) \ni(\rho, \sigma)$ $\rightarrow \rho \sigma \in \widetilde{F}(G)$ is continuous with respect to the topology.

Proof. (i) Clearly we have $\left(\rho ; \Omega_{1} \cup \Omega_{2}, U_{1} \cap U_{2}\right) \subset\left(\rho ; \Omega_{1}, U_{1}\right) \cap\left(\rho ; \Omega_{2}, U_{2}\right)$. Now let $\sigma$ be in $(\rho ; \Omega, U)$. Then the set $\Omega^{\prime}=\left\{\rho(x)^{-1} \sigma(x) \mid x \in \Omega\right\}$ is compact as a continuous image of $\Omega$. Since $\Omega^{\prime}$ is a subset of $U$, we can find a nucleus $U^{\prime}$ so that $\left({ }^{7}\right) \Omega^{\prime} U^{\prime} \subset U$, and we have $\left(\sigma ; \Omega, U^{\prime}\right) \subset(\rho ; \Re, U)$. Therefore the set of all $(\rho ; \Re, U)$ 's defines a topology.

(ii) In order to show that $\widetilde{\mathscr{E}}(G)$ is a Hausdorff space we take two distinct endomorphisms $\rho_{1}$ and $\rho_{2}$ of $G$. Take an element $x$ of $G$ so that $\rho_{1}(x) \neq \rho_{2}(x)$. Then for a nucleus $U$ satisfying $\rho_{1}(x)^{-1} \rho_{2}(x) \notin U^{2}$ we have $\left(\rho_{1} ;\{x\}, U\right) \cap\left(\rho_{2}\right.$; $\{x\}, U)=\varnothing$, where $\{x\}$ denotes the set composed of a single element $x$, and $\varnothing$ is the empty set.

(iii) For a given neighborhood $(\sigma \tau ; \Omega, U)$ of $\sigma \tau$, we take a nucleus $V$ satisfying $\sigma(V) V \subset U$. Then we have $\left.{ }^{8}\right)$

$$
(\sigma ; \tau(\Re) \bar{V}, V)(\tau ; \Re, V) \subset(\sigma \tau ; \Re, U),
$$

(4) On topological groups and especially on locally compact topological groups, see Pontrjagin [14] and Weil [15].

(5) A continuous automorphism is not bicontinuous in general. However if the space of $G$ is a countable union of compact sets, then the continuity of an automorphism implies openness. Cf. Goto [8].

(6) $\{* \mid \cdots\}$ is the set of *'s satisfying $\cdots$.

(7) In general, $\mathfrak{A} \mathfrak{B}=\{a b \mid a \in \mathfrak{A}, b \in \mathfrak{B}\}$ for two subsets $\mathfrak{A}$ and $\mathfrak{B}$ of a semigroup $\mathbb{B}$.

(8) $\bar{V}$ denotes the closure of $V$. 
which implies the continuity of the multiplication.

Hereafter by a topology of $\widetilde{F}(G)$ we mean the topology introduced above. Here we mention two propositions concerning $\widetilde{E}(G)$ without proof, because these are direct consequences of the definition of the topology.

Proposition 2. The mapping $\mu$ from $\widetilde{\mathfrak{F}}(G) \times G$ into $G$ defined by $\mu(\rho, x)$ $=\rho(x)$ is continuous. Moreover the above topology of $\mathbb{E}(G)$ is the weakest of the topologies of $\widetilde{E}(G)$ having the properties in Proposition 1 and making $\mu$ continuous.

Proposition 3. Let $G$ be a locally compact group, and let $N$ be a closed normal subgroup of $G$. Let $\widetilde{\mathfrak{F}}^{N}(G)$ be the subset (semigroup) of $\widetilde{\mathfrak{F}}(G)$ composed of endomorphisms which map $N$ into itself. Let $\sigma$ be in $\widetilde{G}^{N}(G)$. Then $\sigma$ induces an endomorphism $\psi(\sigma)$ of $G / N$, and the mapping $\psi: \widetilde{\mathbb{F}}^{N}(G) \rightarrow \widetilde{\mathbb{E}}(G / N)$ is continuous.

Proposition 4. Let $G$ be a locally compact group generated by a nucleus $W$, then the set of neighborhoods of $\rho$ given by $\{(\rho ; \bar{W}, V) \mid V$ runs over all nuclei $\}$ forms a base of $\rho$ in $\widetilde{E}(G)$.

Proof. Let $(\rho ; \Omega, U)$ be a neighborhood of $\rho$. Then we can find a positive integer $n$ so that $\Re \subset W^{n}$. Since $\bar{W}$ is compact and $\rho$ is continuous, $\rho(\bar{W})$ is compact. Hence we can find a nucleus $V$ so that $V^{n} \subset U$ and $z V=V z$ for $z \in \rho(\bar{W})$.

Let us prove that $(\rho ; \bar{W}, V) \subset(\rho ; \Omega, U)$.

Let $y=x_{1} x_{2} \cdots x_{n}, x_{i} \in \bar{W}$, be an element in $\bar{W}^{n}$, and let $\sigma$ be in $(\rho: \bar{W}, V)$. Since $\rho\left(x_{i}\right)^{-1} \sigma\left(x_{i}\right) \in V$ and $\rho\left(x_{i}\right)^{-1} V=V \rho\left(x_{i}\right)^{-1}$, we have $\rho(y)^{-1} \sigma(y)$ $=\rho\left(x_{n}\right)^{-1} \rho\left(x_{n-1}\right)^{-1} \cdots \rho\left(x_{1}\right)^{-1} \sigma\left(x_{1}\right) \cdots \sigma\left(x_{n}\right) \in V^{n} \subset U$, whence $(\rho ; \bar{W}, V)$ $\subset\left(\rho ; \bar{W}^{n}, U\right) \subset(\rho ; \Omega, U)$.

Next, let us consider some further concepts related to $\widetilde{E}(G)$ which shall be necessary for our purposes later.

$1^{\circ}$. Semigroup of compact subsets of $G$. Let $G$ be a locally compact group, and let $2^{G}$ be the set of all compact nonempty subsets of $G .2^{G}$ forms a semigroup with respect to the product $\Omega_{1} \Omega_{2}=\left\{k_{1} k_{2} \mid k_{1} \in \Omega\right.$ and $\left.k_{2} \in \Omega_{2}\right\}$, (Gleason $[4])$. Let $U$ be a nucleus, and $\Omega$ an element of $2^{G}$. Define the set $(\Omega ; U)$ by $(\Omega ; U)=\left\{\Omega^{\prime} \mid \Omega^{\prime} \in 2^{a}, \Omega^{\prime} \subset \Omega U\right.$ and $\left.\Omega \subset \Omega^{\prime} U\right\}$. Now it is easy to prove the following propositions:

Proposition 5. By considering all ( $\Omega: U$ )'s, where $U$ runs over all nuclei, as a base of the neighborhoods of $\Omega$ in $2^{G}, 2^{G}$ becomes a locally compact Hausdorff space so that the multiplication is continuous.

Proposition 6. The mapping $\nu$ from $\widetilde{\&}(G) \times 2^{G}$ into $2^{G}$ defined by $\nu(\rho, \Omega)$ $=\rho(\Omega)$ is continuous.

$2^{\circ}$. Automorphism group of $G$. Since $\tilde{\mathfrak{A}}(G)$ is a subset of $\widetilde{\mathfrak{F}}(G)$, we may consider the relative topology of $\widetilde{\mathfrak{A}}(G)$ as a subspace of $\widetilde{\mathscr{E}}(G)$, which we shall use as the topology of $\tilde{\mathfrak{A}}(G)$ hereafter, (Goto [7], Hochschild [9], Iwasawa [10] 
and Nomizu and Goto [13]).

To show that $\widetilde{\mathfrak{A}}(G)$ is a topological group under some additional conditions, we need the structure theorem of locally compact groups, and the proof will be given in $\$ 4$.

$3^{\circ}$. Component group (semigroup). Let $G$ be a topological $\operatorname{semigroup}\left({ }^{3}\right)$ with the identity $e$. Then the connected component $G^{0}$ containing $e$ is a closed sub-semigroup. We call $G^{0}$ the component semigroup of $G$. If in particu$\operatorname{lar} G$ is a topological group, then $G^{0}$ becomes a subgroup called the component group of $G$.

We shall use the notation $\mathbb{E}(G)$ for the component semigroup of $\widetilde{\mathscr{F}}(G)$ and $\mathfrak{A}(G)$ for the component semigroup of $\mathfrak{A}(G)$.

$4^{\circ}$. Group of inner automorphisms. Let $G$ be a locally compact group and $a$ an element of $G$. The mapping $\tau_{a}(x)=a^{-1} x a(x \in G)$ defines a bicontinuous automorphism of $G . \tau_{a}$ is called an inner automorphism of $G$. The correspondence $a \rightarrow \tau_{a}$ gives a continuous homomorphism of $G$ into $\tilde{\mathfrak{A}}(G)$, and the kernel coincides with the center of $G$.

We denote the group of all inner automorphisms by $\Im(G)$. If $G$ is connected, then $\Im(G) \subset \mathfrak{A}(G)$. Let $H$ be a subgroup of $G$. The set $\left\{\tau_{a} \mid a \in H\right\}$ forms a subgroup of $\Im(G)$. We shall use the notation $\Im_{G}(H)$ for this group.

2. Lie group case. In $\S 2$ we shall be concerned with $\widetilde{\mathscr{F}}(L)$ where $L$ is a Lie group. Some of the arguments here are quite smilar to those for $\tilde{A}(L)$ in $\S \mathrm{XV}$, Chapter IV of Chevalley: Lie groups I.

Let $\mathfrak{R}$ be an $r$-dimensional Lie algebra over the field of real numbers. A linear homomorphic mapping of $\mathfrak{R}$ into itself is called an endomorphism of $\mathbb{R}$, and the set of all endomorphisms of $\mathfrak{R}$ will be denoted by $\widetilde{E}(\mathfrak{R})$. Let now $G L(r)$ be the semigroup of all linear transformations of $r$-dimensional vector space. Then $\widetilde{\mathbb{F}}(\mathbb{R})$ is a sub-semigroup of $G L(r)$. As the topology of $G L(r)$, we use the usual euclidean one of $r^{2}$-dimensional vector space.

Let $e_{1}, e_{2}, \cdots, e_{r}$ be a basis of $\mathbb{R}$. Assume the commutator multiplication of $\mathfrak{R}$ is expressed by $\left[e_{i}, e_{j}\right]=\sum_{s} \gamma_{i j}^{s} e_{s}$ with respect to the basis, where $\gamma_{i j}^{s}$ 's are real numbers called structure constants. Let $\sigma$ be a linear transformation of the vector space $\mathfrak{l}$ so that $\sigma\left(e_{i}\right)=\sum_{u} \xi_{i}^{u} e_{u}$. If $\sigma \in \widetilde{F}(\mathfrak{\&})$ then we have $\left[\sigma\left(e_{i}\right), \sigma\left(e_{j}\right)\right]=\sigma\left(\left[e_{i}, e_{j}\right]\right)$, and vice versa. The condition that a linear transformation $\sigma$ be an endomorphism is written in the form

$$
\sum_{u, v} \xi_{i}^{u} \xi_{j}^{v} \gamma_{u v}^{k}=\sum_{s} \gamma_{i j}^{*} \xi_{s}^{k}
$$

Therefore $\widetilde{F}(\mathbb{R})$ is an algebraic set and is closed in $G L(r)$.

Now let $L$ be a connected Lie group, and let $\&$ be the Lie algebra of $L$. We can identify a sufficiently small nucleus of $L$ with a neighborhood of 0 in $\&$ by taking a canonical system of coordinates of the first kind (Pontrjagin [14]), and for any local endomorphism of $L$ we have a corresponding endomorphism of $\mathfrak{R}$. Hence for any endomorphism $\sigma$ of $L$ we have the correspond- 
ing endomorphism $\psi(\sigma)$ of $\mathfrak{R}$. The correspondence $\sigma \rightarrow \psi(\sigma)$ is clearly a continuous homomorphism, and since $L$ is generated by any nucleus, the homomorphism $\psi$ is one-to-one.

In particular, let $L$ be a connected simply connected Lie group. Then any local endomorphism generates an endomorphism of $L$, and we obtain a bicontinuous isomorphism $\widetilde{夭}(L) \cong \widetilde{\mathscr{G}}(\Omega)$.

Next, let us consider a connected Lie group $L$ which is not necessarily simply connected, and let $\tilde{L}$ be the universal covering group of $L$. Then there exists a discrete normal, hence central, subgroup $F$ of $\widetilde{L}$, which is isomorphic to the fundamental group of the space of $L$, so that $\tilde{L} / F \cong L$. Consider the sub-semigroup $\tilde{\mathscr{E}} F(\tilde{L})$ of $\tilde{\mathscr{E}}(\tilde{L})=\{\sigma \mid \sigma \in \widetilde{\mathscr{E}}(\tilde{L}), \sigma(F) \subset F\}$. Then $\widetilde{\mathscr{F}}^{F}(\tilde{L})$ is clearly a closed sub-semigroup of $\widetilde{\mathfrak{E}}(\tilde{L})$, and an element of $\widetilde{\mathfrak{F}}^{F}(\tilde{L})$ induces an endomorphism $\phi(\sigma)$ of $\tilde{L} / F \cong L$. On the other hand for a given endomorphism $\sigma^{\prime}$ of $L$, we have a corresponding local endomorphism of $\tilde{L}$, which induces $\sigma$ in $\widetilde{\mathscr{E}}(\tilde{L})$ so that $\sigma(F) \subset F$ and $\phi(\sigma)=\sigma^{\prime}$. So $\widetilde{\mathscr{E}}(L)$ is naturally identified with $\widetilde{G}^{F}(\widetilde{L})$, which is closed in $\widetilde{\mathscr{E}}(\tilde{L})$. Thus we get

Proposition 7. Let $L$ be an r-dimensional connected Lie group. Then we have a bicontinuous isomorphism of $\widetilde{\xi}(L)$ onto a closed sub-semigroup of $G L(r)$. Hence $\widetilde{E}(L)$ is locally compact.

REMARK. Although $\tilde{\mathfrak{A}}(L)$ is a Lie group, $\widetilde{\mathfrak{F}}(L)$ is not locally euclidean in general.

In a manner analogous to the proof of Proposition 7, we have the following

Proposition 8. Let $L$ be a connected Lie group of $r$-dimension, and let $D$ be a discrete normal subgroup of $L$. Let $\widetilde{ङ}_{D}(L)$ be the sub-semigroup composed of all endomorphisms of $L$ which leave every element of $D$ fixed, and let $\mathbb{E}_{D}(L)$ be the component semigroup of $\widetilde{E}_{D}(L)$. Then $\widetilde{E}_{D}(L)$, and accordingly $\mathbb{E}_{D}(L)$ also, is a closed sub-semigroup of $G L(r)$.

Let $\sigma$ be an endomorphism of a connected Lie group $L$, and let $|\sigma|$ be the determinant of the corresponding linear transformation. Then the mapping $\sigma \rightarrow|\sigma|$ clearly defines a continuous homomorphism of $\widetilde{E}(L)$ into the multiplicative semigroup of real numbers. Since $|\sigma| \neq 0$ implies $\sigma$ is a local automorphism and vice versa, we have the following

Proposition 9. Let $\sigma$ be an endomorphism of a connected Lie group L. Then $\sigma$ is onto if and only if $|\sigma| \neq 0$.

In general, an endomorphism of $L$ onto itself is not an automorphism. However it is obviously true for simply connected groups, and is also true in the following case.

Proposition 10. Let $L$ be a connected Lie group whose center is discrete. Then any onto endomorphism is an automorphism. 
Proof. Let $\sigma$ be an endomorphism of $L$ onto $L$, and let $D$ be the kernel of the homomorphism $\sigma$. Then $L \cong L / D$. So $\operatorname{dim} L=\operatorname{dim} L / D$, whence $D$ is discrete. Therefore $D$ is contained in the center $C$ of $L$. Now, it is clear that $C / D$ is the center of $L / D$. Hence $C$ is isomorphic with $C / D$. On the other hand, since $C$ has a finite system of generators (Goto [5]), $C$ cannot be isomorphic with any proper factor group. Hence $D=e\left({ }^{9}\right)$.

Corollary. Let $S$ be a connected semi-simple Lie group. Then $\mathbb{E}(S)=\mathfrak{A}(S)$, which is open in $\widetilde{E}(S)$.

Proof. It is well-known $\left({ }^{10}\right)$ that $|\sigma|= \pm 1$ for $\sigma \in \tilde{\mathfrak{A}}(S)$. On the other hand a semi-simple Lie group has a discrete center. Hence $|\sigma|$ can take only the values \pm 1 or 0 for $\sigma \in \widetilde{\mathbb{F}}(S)$. Since the mapping $\sigma \rightarrow|\sigma|$ is continuous and $|1|=1, \mathbb{E}(S) \subset\{\sigma|| \sigma \mid=1\} \subset \widetilde{\mathscr{A}}(S)$, which is open and closed in $\widetilde{E}(S)$. Hence $\mathfrak{E}(S)=\mathfrak{A}(S)$. On the other hand $\mathfrak{A}(S)$ is known to be open in $\mathfrak{A}(S)\left({ }^{10}\right)$. $\widetilde{⿷}(L)$.

Proposition 11. Let $L$ be a connected Lie group. Then $\widetilde{\mathfrak{A}}(L)$ is open in

Proof. (i) Let $Z$ be a compact connected abelian Lie group. Take a canonical coordinate system of the first kind in $Z$ so that $\left(x_{i}\right)\left(y_{i}\right)=\left(x_{i}+y_{i}(\bmod 1)\right)$. Then with respect to the coordinate system, any endomorphism of $Z$ has a matrix with integer coefficients. So $\widetilde{\mathfrak{F}}(Z)$ is discrete. Hence $\widetilde{\mathfrak{A}}(Z)$ is, of course, open in $\widetilde{\mathfrak{G}}(Z)$.

Let now $L$ be a semi-simple Lie group or a vector group of finite dimension. Then $\widetilde{\mathfrak{A}}(L)$ coincides with $\widetilde{\mathfrak{F}}^{\prime}(L)=\{\sigma|\sigma \in \widetilde{\mathfrak{F}}(L),| \sigma \mid \neq 0\}$.

(ii) Let $L$ be a connected Lie group and let $\widetilde{F}^{\prime}(L)$ be the sub-semigroup of $\widetilde{\mathbb{E}}(L)$ composed of all onto endomorphisms. $\widetilde{\mathbb{F}}^{\prime}(L)$ is clearly open in $\widetilde{\mathbb{E}}(L)$.

Now in $L$ we shall construct a sequence of closed normal subgroups

$$
L=L_{0} \supset L_{1} \supset \cdots \supset L_{n}=e,
$$

so that

$$
\tilde{\mathbb{F}}^{\prime}(L) L_{i} \subset L_{i},
$$

and each factor group $L_{i} / L_{i+1}$ is semi-simple, compact abelian, or is a vector group. For that we take as $L_{1}$ the radical (maximal connected closed solvable normal subgroup), take as $L_{3}$ the closure of the commutator subgroup of $L_{1}$, and the closure of the commutator subgroup of $L_{3}$ as $L_{5}$, and so on. $L_{2 m}$ will be given so that $L_{2 m} / L_{2 m+1}$ is the maximal compact subgroup of $L_{2 m-1} / L_{2 m+1}$ for $m=1,2,3, \cdots$.

Then since any element of $\tilde{\mathbb{F}}^{\prime}(L)$ is a local automorphism of $L$ we have $\widetilde{F}^{\prime}(L) L_{1} \subset L_{1}$. Then it is clear that $\mathbb{F}^{\prime}(L) L_{i} \subset L_{i}$ for $i=2,3, \cdots$ Also, from

( $\left.{ }^{9}\right)$ We may use the notation $e$ sometimes instead of $\{e\}$.

(10) It is known that $\mathfrak{A}(S)=\mathfrak{Y}(S)$ and $\mathfrak{Y}(S)$ has a finite index in $\mathfrak{A}(S)$. (See Gantmacher [2]). Since $\Im(S)$ is a connected Lie group with no nontrivial abelian factor groups, $|\sigma|=1$ for $\sigma \in \Im(S)$. So $|\sigma|$ is a representation of the finite group $\widetilde{\mathscr{A}}(S) / \Im(S)$ into the multiplicative group of real numbers. Hence $|\sigma|= \pm 1$. 
the construction, $L_{0} / L_{1}$ is semi-simple, $L_{2 m-1} / L_{2 m}$ is a vector group, and $L_{2 m} / L_{2 m+1}$ is compact and abelian.

(iii) Let $\sigma$ be an endomorphism of $L$ onto itself. Then $\sigma$ induces an endomorphism $\sigma_{i}$ of $L_{i} / L_{i+1}$, and $\sigma$ is an automorphism if and only if all $\sigma_{i}$ 's are so. Since the mapping $\sigma \rightarrow \sigma_{i}$ is continuous by Proposition 3, the problem is reduced to the special cases of (i).

Corollary. Let $L$ be a connected Lie group. If the radical of $L$ is simply connected, then $\tilde{\mathfrak{E}}^{\prime}(L)=\widetilde{\mathfrak{A}}(L)$.

Proof. The Corollary is true for vector groups and semi-simple Lie groups. On the other hand if the radical of $L$ is simply connected, then in the sequence of factor groups in the proof above, each $L_{i} / L_{i+1}$ can be either a vector group or a semi-simple group.

3. Compact group case $\left({ }^{11}\right)$. In $\S 3$ the following theorem and some related results shall be obtained.

THEOREM 1. Let $G$ be a compact group. Then $\&(G)=\mathfrak{A}(G)$.

Before proving the theorem, we shall obtain the following preliminary proposition.

Proposition 12. Let $G$ be a locally compact group, and $U$ a nucleus of $G$. If $U$ contains a closed subgroup $K$ containing all subgroups of $G$ in $U$, then

$$
\widetilde{\mathfrak{F}}^{K}(G)=\{\sigma \mid \sigma \in \widetilde{\mathfrak{F}}(G), \sigma(K) \subset K\}
$$

forms an open and closed sub-semigroup of $\widetilde{E}(G)$.

Hence we have $\&(G) \subset \widetilde{F} K(G)$.

Proof. Let $T$ be the set of all compact subgroups of $G$, and let $T^{\prime}$ be the set of all closed subgroups of $K$. Then $T$ is a closed subset of $2^{a}$, and $T^{\prime}$ is clearly an open and compact subset of $T$. Hence by Proposition 5

$$
\mathfrak{F}^{K}(G)=\left\{\sigma \mid \sigma \in \widetilde{\mathscr{E}}(G), \sigma\left(T^{\prime}\right) \subset T^{\prime}\right\}
$$

is closed and open.

Proof of Theorem 1. (i) Let $L^{0}$ be a compact connected Lie group, let $S$ be the commutator subgroup of $L^{0}$, and let $Z$ be the component group of the center of $L^{0}$. Then $S$ is a closed semi-simple normal subgroup, and $L^{0}=S Z$ is a locally direct decomposition.

Let $\sigma$ be an endomorphism of $L^{0}$. Since any endomorphism leaves the commutator subgroup fixed, $\sigma$ induces an endomorphism of $S$. If in particular $\sigma \in \mathbb{E}\left(L^{0}\right)$, then the induced endomorphism $\sigma_{1}$ of $S$ is an automorphism by the corollary to Proposition 10.

Since $\left({ }^{12}\right)[Z, S]=e$, we have $[\sigma(Z), \sigma(S)]=e$, namely $[\sigma(Z), S]=e$. There-

(11) About the theory of compact groups, see Pontrjagin [14] and Weil [15].

(12) Let $\mathfrak{A}$ and $\mathfrak{B}$ be nonempty subsets of a group $\mathfrak{B}$. [ $[\mathfrak{A}, \mathfrak{B}]$ denotes the subgroup generated by $\left\{a b a^{-1} b^{-1} \mid a \in \mathfrak{A}\right.$ and $\left.b \in \mathfrak{B}\right\}$. For example [(S), (S)] is the commutator subgroup of $\mathbb{B}$. 
fore $\left[\sigma(Z), L^{0}\right]=e$. On the other hand $\sigma(Z)$ is connected. Hence $\sigma(Z) \subset Z$. So $\sigma$ induces an endomorphism of $Z$. By using the connectedness of $\mathbb{E}\left(L^{0}\right)$ again, we have $\sigma=1$ on $Z$.

These show that $\sigma \in \tilde{\mathfrak{A}}\left(L^{0}\right)$, so $\sigma \in \mathfrak{A}\left(L^{0}\right)$.

(ii) Let $L$ be a compact Lie group, and $L^{0}$ the component group of $L$. Let $\sigma$ be an endomorphism of $L$. Then $\sigma$ leaves $L^{0}$ invariant and induces an endomorphism $\psi(\sigma)$ of $L^{0}$. Since $\psi$ is continuous, we have $\psi(\xi(L)) \subset \mathbb{E}\left(L^{0}\right)$ $=\mathfrak{A}\left(L^{0}\right)$.

Let $L=a_{1} L^{0}+\cdots+a_{m} L^{0}$ be the coset decomposition of $L$ with respect to $L^{0}$. Then the set $\left\{\sigma \mid \sigma \in \widetilde{\mathscr{F}}(L), \sigma\left(a_{1}\right) \in a_{1} L^{0}, \cdots, \sigma\left(a_{m}\right) \in a_{m} L^{0}\right\}$ forms an open and closed sub-semigroup of $\widetilde{E}(L)$, and contains $\mathbb{E}(L)$. Hence $\sigma\left(a_{i}\right) \in a_{i} L^{0}$ for $\sigma \in \notin(L)$.

Therefore $\sigma$ is an automorphism of $L$.

(iii) Let $G$ be a compact group. Then we can find a set $\left\{U_{\alpha}\right\}$ of nuclei, forming a base at $e$, so that

$$
U_{\alpha}=L_{\alpha}^{\prime} \times N_{\alpha},
$$

namely $U_{\alpha}=L_{\alpha}^{\prime} N_{\alpha}, L_{\alpha}^{\prime} \cap N_{\alpha}=e,\left[L_{\alpha}^{\prime}, N_{\alpha}\right]=e$, where $N_{\alpha}$ is a closed normal subgroup of $G, L_{\alpha}=G / N_{\alpha}$ is a Lie group, and $L_{\alpha}^{\prime}$ is a local Lie group isomorphic to a nucleus of $L_{\alpha}$. We may assume that $L_{\alpha}^{\prime}$ does not contain any subgroup other than $e$. Then $U_{\alpha}$ and $N_{\alpha}$ clearly satisfy the assumptions in Proposition 12. Therefore an element $\sigma$ in $\&(G)$ induces an endomorphism $\sigma_{\alpha}$ of $L_{\alpha}$, which is obviously in $\&\left(L_{\alpha}\right)=\mathfrak{A}\left(L_{\alpha}\right)$.

Let $D$ be the kernel of $\sigma$. Since $\sigma_{\alpha}$ is one-to-one on $L_{\alpha}, D$ should be contained in $N_{\alpha}$. On the other hand $\cap N_{\alpha} \subset \cap U_{\alpha}=e$. Hence $D=e$, that is, $\sigma$ is one-to-one.

Now, let us show that $\sigma$ is an onto mapping. Let $a$ be an element of $G$, and let $M_{\alpha}$ be the set of all elements $a_{\alpha}$ so that $\sigma\left(a_{\alpha}\right) \in a N_{\alpha}$. Since $\sigma_{\alpha}$ is an automorphism, $M_{\alpha}$ is not empty. Moreover, for given $\alpha_{1}, \alpha_{2}, \cdots, \alpha_{n}$, we can find $\alpha_{0}$ so that $\bigcap_{i=1}^{n} U_{\alpha_{i}} \supset U_{\alpha_{0}}$. For such $\alpha_{0}$ we have $N_{\alpha_{1}} \cap \cdots \cap N_{\alpha_{n}} \supset N_{\alpha_{0}}$ whence $M_{\alpha_{1}} \cap \cdots \cap M_{\alpha_{n}} \supset M_{\alpha_{0}}$. Therefore the set $\left\{M_{\alpha}\right\}$ of compact sets has the finite intersection property. So we can take $b$ in $\cap M_{\alpha}$. Then $\sigma(b)=a N_{\alpha}$ for all $\alpha$. Hence $\sigma(b)=a$.

Corollary 1. Let $G$ be a compact group and $G^{0}$ the component group of $G$. Then

$$
\mathfrak{F}(G)=\mathfrak{A}(G) \cong \Im\left(G^{0}\right) \cong \Pi_{\alpha} S_{\alpha},
$$

where $\Im\left(G^{0}\right)$ is the group of all inner automorphisms of $G$, induced by elements of $G^{0}$ and $S_{\alpha}$ 's are compact connected simple Lie groups with no centers (Iwasawa [10] and Goto [7]).

Next, let us consider a locally compact connected group $G$. By the radical of $G$ we mean also the uniquely determined maximal solvable closed con- 
nected normal subgroup $R$ (Gleason [3], Iwasawa [10] and Matsushima [11]). If $R$ is compact, we can find a closed connected normal semi-simple Lie group $L$ and a compact connected normal subgroup $K$ so that $G=L K$, $[L, K]=e$, and $L \cap K$ is a finite group (Goto [6]). Then the following corollary is a direct consequence of Proposition 10 and Theorem 2.

COROLLARY 2. Let $G$ be a locally compact connected group whose radical is compact. Then $\mathfrak{E}(G)=\mathfrak{A}(G)=\Im(G)$.

Here the last equality follows from the corresponding theorem for connected semi-simple Lie groups $\left({ }^{10}\right)$.

4. Automorphism group. First, let us remember the already known structure theorem of locally compact groups.

StRUCTURE TheOREm. Let $G$ be a locally compact group. Then we can find a base at e, composed of nuclei of the form $W=L_{l}^{*} \times K$, where $L_{l}{ }^{*}$ is a local Lie group, and $K$ is a compact subgroup (Montgomery-Zippin [12], Iwasawa [10] and Yamabe $[16 ; 17])$.

Let us consider the group $L^{*}$ which is generated by $L_{l}^{*}$ in $G$. Then we can find a uniquely determined connected Lie group $L$ which maps continuously and isomorphically onto $L^{*}$. The subgroup $L^{*} K$ is open in $G$.

If in particular $G$ is connected, then $K$ is a normal subgroup and $G=L^{*} K$, $\left[L^{*}, K\right]=e$. Denote by $D^{*}$ the intersection of $L^{*}$ and $K$, and let $D$ be the inverse image of $D^{*}$ in $L$. Then the fact $L_{l}{ }^{*} \cap K=e$ implies that $D$ is a discrete normal, accordingly central, subgroup of $L$.

Let us call a local decomposition of $G$, as in the structure theorem, a canonical decomposition, including the notions of $L, L^{*}, D$ and $D^{*}$, in the connected case.

Theorem 2. Let $G$ be a locally compact group, and let $W_{0}=L_{0}^{*} \times K$ be a local canonical decomposition of $G$. If $G$ is generated by $W_{0}$, then $\mathfrak{A}(G)$ is a topological group, (i.e., $\sigma^{-1}$ is a continuous function on $\mathfrak{A}(G)$ ).

Proof. (i) Let $L$ be a connected Lie group and $L_{0}$ a nucleus of $L$. As we saw, $\widetilde{\mathfrak{E}}(L)$ has a linear representation obtained by taking a canonical system of coordinates of the first kind. Let $L_{1}$ and $L_{2}$ be open spheres of radius $r_{1}$ and $r_{2}$, respectively, with respect to the coordinate system. If $r_{1}<r_{2}$ and if $r_{2}$ is sufficiently small, then we can find a neighborhood $\mathfrak{U}$ of the identity 1 of $\widetilde{\mathfrak{F}}(L)$ so that $\mathfrak{U} \ni \tau$ implies $L_{1} \subset \tau\left(L_{2}\right) \subset L_{0}$. Since $\widetilde{\mathfrak{A}}(L)$ is open in $\widetilde{\mathfrak{F}}(L)$ by Proposition 11 we may take $\mathfrak{U}$ in $\widetilde{\mathfrak{A}}(L)$.

(ii) Let $G$ be a locally compact group satisfying the assumptions in Theorem 2. Since $G$ is generated by $W_{0}, K$ is a normal subgroup of $G$. We use the notation $L$ here for the factor group $G / K$. By (i) we get nuclei $L_{1}^{*}$ and $L_{2}^{*}$ of $L_{0}^{*}$ so that $L_{1}^{*} \subset \tau\left(L_{2}^{*}\right) \subset L^{*}$ for $\tau \in \mathfrak{U}$ where $\mathfrak{U}$ is a neighborhood of 1 in $\widetilde{F}(L)$. The nucleus $W_{1}=L_{1}^{*} \times K$ also generates $G$. 
Let us consider the sub-semigroup $\widetilde{\mathfrak{C}}^{K}(G)$ of $\widetilde{\mathfrak{F}}(G)$ composed of all endomorphisms leaving $K$ invariant. Then we have a continuous homomorphism $\psi$ from $\widetilde{\mathfrak{F}}^{K}(G)$ into $\widetilde{\mathfrak{F}}^{(}(L)$. Since $\widetilde{\mathfrak{F}}^{K}(G)$ is open in $\mathbb{E}(G)$, by Proposition 12 , and any neighborhood of 1 contains a neighborhood of the form $\left(1 ; \bar{W}_{1}, V\right)$, we may find a nucleus $V$ of $G$ so that $\psi\left(\left(1 ; \bar{W}_{1}, V\right)\right) \subset \mathfrak{U}$.

We use the notation $[1 ; \Re, V]$ for the intersection of $(1 ; \Re, V)$ and $\tilde{\mathfrak{A}}(G)$. Since $\mathfrak{U} \subset \tilde{\mathfrak{A}}(L)$, any element $\sigma$ of $\left[1 ; W_{1}, V\right]$ induces an automorphism of $L$, whence $\sigma(K)=K$.

Let $\sigma$ be in $\left[1 ; W_{2}, V\right]$ where $W_{2}=L_{2}{ }^{*} \times K$. Then for $x \in \bar{W}_{2}, x^{-1} \sigma(x) \in V$, namely $\left(\sigma^{-1}(y)\right)^{-1} y \in V$ for $y \in \sigma\left(\bar{W}_{2}\right)$. Since $V=V^{-1}$, we have $y^{-1}\left(\sigma^{-1}(y)\right) \in V$ for $y \in \sigma\left(\bar{W}_{2}\right)$. On the other hand since $\psi(\sigma) \in \mathfrak{U}$ we have $L_{1}^{*} \subset \psi(\sigma) L_{2}{ }^{*} \subset L_{0}^{*}$. Therefore $\sigma\left(W_{2}\right)=\sigma\left(L_{2}^{*}\right) \times \sigma(K)=\sigma\left(L_{2}^{*}\right) \times K=\psi(\sigma) L_{2}^{*} \times K \supset L_{1}^{*} \times K=W_{1}$. Hence for $y \in \bar{W}_{1}$, we have $y^{-1}\left(\sigma^{-1}(y)\right) \in V$, namely

$$
\left[1 ; \bar{W}_{2}, V\right]^{-1} \subset\left[1 ; \bar{W}_{1}, V\right]
$$

which proves the continuity of $\sigma^{-1}$.

5. Structure of $\&(G)$ of a locally compact connected group $G$. Let $G=L^{*} K$ be a canonical decomposition of a locally compact connected group $G$. In $\S 5$ we will study the structure of $\mathbb{E}(G)$ with respect to the decomposition (Goto $[7])$.

Let $K^{0}$ be the component group of $K$. Then $K^{0}$ is a normal subgroup. Let $k_{1}$ and $k_{2}$ be elements of $K^{0}$. If $k_{1}^{-1} x k_{1}=k_{2}^{-1} x k_{2}$ for every $x \in K$, then $k_{1} k_{2}^{-1}$ is contained in the center of $K^{0}$. On the other hand, in a connected topological group any compact abelian normal subgroup is central, because $\mathfrak{A}(Z)$ for a compact abelian group $Z$ consists only of the identity (Iwasawa [10]). Hence $k_{1} k_{2}^{-1}$ is contained in the center of $G$. Let $\Im_{G}\left(K^{0}\right)$ and $\Im_{K}\left(K^{0}\right)$ be the subgroups of $\widetilde{\mathfrak{A}}(G)$ and $\widetilde{\mathfrak{A}}(K)$ composed of all inner automorphisms induced by elements of $K^{0}$ respectively. Then by the above argument we have a natural bicontinuous isomorphism between $\Im_{G}\left(K^{0}\right)$ and $\Im_{K}\left(K^{0}\right)$.

Let $\sigma$ be an element in $\varangle(G)$. Then by Proposition 12 we have $\sigma(K) \subset K$. Hence $\sigma$ induces an endomorphism of $K$. Since $\&(K)$ is connected, $\sigma \in \mathbb{E}(K)$ $=\Im_{K}\left(K^{0}\right)$ on $K$ by Theorem 2. So we obtain a natural homomorphism $\phi$ from $\mathscr{E}(G)$ in to $\Im_{G}\left(K^{0}\right)$. $\phi$ is clearly an onto mapping.

Let now $\mathfrak{B}$ be the set of all elements of $\mathfrak{E}(G)$ which go to the identity by $\phi$ :

$$
\mathfrak{B}=\{\sigma \mid \sigma \in \mathbb{F}(G), \phi(\sigma)=1\}=\left\{\sigma \mid \sigma \in \mathcal{E}(G), \sigma(x)=x \text { for all } x \in K^{0}\right\} .
$$

We shall prove that $\Im_{G}\left(K^{0}\right)$ and $\mathfrak{B}$ are elementwise commutative. Let $\rho$ be in $\Im_{G}\left(K^{0}\right)$. Then there is an element $a$ of $K^{0}$ such that $\rho(x)=a^{-1} x a$ for $x \in G$. Now let $\sigma$ be in $\mathfrak{B}$. Then for $x$ in $G$ we have

$$
(\sigma \rho)(x)=\sigma\left(a^{-1} x a\right)=\sigma(a)^{-1} \sigma(x) \sigma(a)=a^{-1} \sigma(x) a=(\rho \sigma)(x),
$$

namely $\sigma \rho=\rho \sigma$.

Let $\sigma$ be an element in $\&(G)$. Then

$$
\psi(\sigma)=\phi(\sigma)^{-1} \sigma \in \mathfrak{B},
$$


because $\phi(\psi(\sigma))=\phi\left(\phi(\sigma)^{-1}\right) \phi(\sigma)=\phi(\sigma)^{-1} \phi(\sigma)=1$. So $\sigma$ has a decomposition

$$
\sigma=\phi(\sigma) \psi(\sigma) \text { where } \phi(\sigma) \in \Im_{G}\left(K^{0}\right) \text { and } \psi(\sigma) \in \mathfrak{B} \text {. }
$$

Let $\sigma=\rho \tau\left(\rho \in \Im_{G}\left(K^{0}\right), \tau \in \mathfrak{B}\right)$ be another decomposition. Then $\phi(\sigma)=\phi(\rho) \phi(\tau)$ $=\phi(\phi(\sigma)) \phi(\psi(\sigma))$, so $\rho=\phi(\sigma)$. Hence $\tau=\rho^{-1} \sigma=\psi(\sigma)$. Therefore the decomposition $\left(^{*}\right)$ is unique.

Since $\phi$ and $\psi$ are clearly both continuous, the mapping $\theta$ :

$$
\leftleftarrows(G) \ni \sigma \rightarrow \theta(\sigma)=(\phi(\sigma), \psi(\sigma)) \in \Im_{G}\left(K^{0}\right) \times \mathscr{B}
$$

is continuous. Moreover $\theta$ is an onto mapping because $\&(G)$ contains both $\Im_{G}\left(K^{0}\right)$ and $\mathfrak{B}$.

Next, let us prove the continuity of $\theta^{-1}$. Let $(\sigma ; \Re, U)$ be a neighborhood of $\sigma$ in $\&(G)$. Take nuclei $U_{1}$ and $U_{2}$ of $G$ so that $U_{1}^{2} \subset U$ and $\phi(\sigma)\left(U_{2}\right) \subset U_{1}$. Then it is easy to show that

$$
\theta(\sigma ; \Re, U) \supset\left(\phi(\sigma) ;(\psi(\sigma) \Re) \bar{U}_{2}, U_{1}\right) \times\left(\psi(\sigma) ; \Re, U_{2}\right),
$$

which implies the openness of $\theta$.

Thus we get the following theorem.

TheOREM 3. Let $G=L^{*} K$ be a canonical decomposition of a locally compact connected group $G$, and let $K^{0}$ be the component group of $K$. Then $\mathbb{E}(G)$ is a topological direct product of $\mathfrak{A}$ and $\mathfrak{B}$ :

$$
\mathfrak{E}(G)=\mathfrak{A} \times \mathfrak{B},
$$

where $\mathfrak{A}=\Im_{G}\left(K^{0}\right)=$ the group of inner automorphisms induced by $K^{0}$, and $\mathfrak{B}=$ semigroup $\left\{\sigma \mid \sigma \in \mathfrak{E}(G), \sigma(x)=x\right.$ for all $\left.x \in K^{0}\right\}$.

Now, about the structure of the semigroup $\mathfrak{B}$, we can prove the following theorem.

Theorem 4. In Theorem 3 ,

(1) we have a bicontinuous isomorphism from $\mathfrak{E}_{D}(L)$ into $\mathfrak{B}$ :

$$
f\left(\mathfrak{F}_{D}(L)\right) \subset \mathfrak{B} .
$$

(2) If $L^{*}$ is mapped into itself by $\&(G)$, then

$$
f\left(\xi_{D}(L)\right)=\mathfrak{B}
$$

and $\mathbb{E}(G)$ is locally compact.

Proof. (1) Let $x$ be an element of $L$. We use the notation $x^{*}$ for the corresponding element of $L^{*}$. Let $\sigma$ be in $\mathbb{E}_{D}(L)$. Define the function $f$ by

$$
\left\{\begin{array}{lr}
f(\sigma) x^{*}=(\sigma(x))^{*} & x^{*} \in L^{*}, \\
f(\sigma) y=y & y \in K,
\end{array}\right.
$$

then $f(\sigma)$ can easily be extended uniquely into a homomorphism of $G=L^{*} K$ 
into itself. Since $f(\sigma)$ is continuous on a nucleus of $G, f(\sigma)$ is an endomorphism of $G$. Thus we get a homomorphism $f$ from $\mathcal{E}_{D}(L)$ into $\mathbb{E}(G)$. Clearly $f$ is oneto-one.

Now it is enough for our purposes to show the bicontinuity of $f$.

Take a neighborhood $\left(f(\sigma) ; \bar{L}_{l}^{*} \times K, U\right)$ of $f(\sigma)$. Then we can find a nucleus $V$ of $L$ so small that $k^{-1} V^{*} k \subset U$ for any $k$ in $K$. If $\rho \in\left(\sigma ; \bar{L}_{l}, V\right)$, then $\sigma(x)^{-1} \rho(x) \in V$ for $x \in \bar{L}_{l}$. Hence for any $x^{*} \in \bar{L}_{l}{ }^{*}, k \in K$,

$$
\begin{aligned}
\left((f(\sigma))\left(x^{*} k\right)\right)^{-1}(f(\rho))\left(x^{*} k\right) & =(f(\sigma)(k))^{-1}\left(f(\sigma)\left(x^{*}\right)\right)^{-1}\left(f(\rho)\left(x^{*}\right)\right)(f(\rho)(k)) \\
& =k^{-1}\left(\sigma(x)^{-1} \rho(x)\right)^{*} k \in k^{-1} V^{*} k \subset U,
\end{aligned}
$$

namely $f\left(\sigma ; \bar{L}_{l}, V\right) \subset\left(f(\sigma) ; \bar{L}_{l}^{*} \times K, U\right)$, which implies the continuity of $f$.

Next, let $(\sigma ; \Omega, U)$ be a neighborhood of $\sigma$ in $\xi_{D}(L)$. Then there exists a nucleus $L_{l}^{*} \times K$ in $G$ such that $L_{l} \subset U$. Take any element $\rho$ of $\mathbb{F}_{D}(L)$ such that $f(\rho) \in\left(f(\sigma), K^{*}, L_{l}^{*} \times K\right)$. Then for $x \in \Omega,\left(f(\sigma)\left(x^{*}\right)\right)^{-1}\left(f(\rho)\left(x^{*}\right)\right)$ $=\left(\sigma(x)^{-1} \rho(x)\right)^{*} \in L_{l}^{*} \times K$, whence $\sigma(x)^{-1} \rho(x) \in U$, namely $\rho \in(\sigma ; \Omega, U)$. This shows that $f^{-1}$ is continuous.

(2) If $\mathbb{E}(G) L^{*} \subset L^{*}$, then every element of $\mathbb{E}(G)$ induces an endomorphism of $L^{*}$. On the other hand every element of $\&(G)$ induces an inner automorphism of $K$, and leaves every element of $D^{*}$ fixed. So we can define a homomorphism $f^{\prime}$ from $\mathbb{E}(G)$ into $\widetilde{\mathfrak{E}}_{D}(L) . f^{\prime}$ is clearly one-to-one on $\mathfrak{B}$, and coincides with $f^{-1}$. Therefore $f\left(\mathbb{E}_{D}(L)\right)=\mathfrak{B}$.

Since $L$ is a connected Lie group, $\mathfrak{E}_{D}(L)$ is locally compact by Proposition 8 , and so is $\mathfrak{B}$. Since $\mathfrak{A}$ is a compact group, $\mathfrak{E}(G)=\mathfrak{A} \times \mathfrak{B}$ is locally compact.

REMARK. $\&(G) L^{*} \subset L^{*}$ is not valid in general. However we may prove the following

Theorem 5. Let $G=L^{*} K$ be a canonical decomposition of a locally compact connected group. Then $L^{*}$ is mapped into itself by $\mathbb{E}(G): \mathbb{E}(G) L^{*} \subset L^{*}$, if either

(a) the center of $K$ is totally disconnected, or

(b) $L$ is perfect (i.e. $[L, L]=L)$.

Proof. Let $\sigma$ be an element of $\left(\xi(G)\right.$. We may find a connected nucleus $L_{\rho}$ of $L$ such that

$$
\sigma\left(L_{\rho}^{*}\right) \subset L_{l}^{*} \times K
$$

where $L_{\rho}^{*}$ denotes the image of $L_{\rho}$ in $L^{*}$. Then for $x \in L_{\rho}^{*}$ we have a continuous decomposition

$$
\sigma(x)=l(x) k(x) \quad \text { where } \quad l(x) \in L_{l}^{*}, \quad k(x) \in K .
$$

Jet $y$ be an element of $K$. Since $x y=y x$, we have $\sigma(x) \sigma(y)=\sigma(y) \sigma(x)$, and therefore

$$
l(x) k(x) \sigma(y)=\sigma(y) l(x) k(x)=l(x) \sigma(y) k(x),
$$

whence $k(x) \sigma(y)=\sigma(y) k(x)$. Since $\sigma$ induces an automorphism of $K, \sigma(y)$ can be an arbitrary element of $K$. Hence $k(x)$ is contained in the center of $K$. 
Now we consider the two cases (a) and (b) separately:

(a) Because $\left\{k(x) \mid x \in L_{\rho}^{*}\right\}$ is connected, and is contained in the totally disconnected center of $K$,

(b) because $k(x)$ gives a continuous homomorphism from a perfect local Lie group $L_{\rho}^{*}$ into a commutative group,

$k(x)=e$ in both cases.

Namely $\sigma\left(L_{l}^{*}\right) \subset L_{l}^{*}$, whence $\sigma\left(L^{*}\right) \subset L^{*}$.

Corollary. Let $G$ be a locally compact connected group. If $G$ is perfect or the center of $G$ is totally disconnected, then $\&(G)$ is locally compact.

Proof. (a) If $G$ is perfect, so is the factor group $G / K$. On the other hand, every connected locally isomorphic group of a connected perfect Lie group is perfect. Since $L$ is locally isomorphic with $G / K, L$ is perfect.

(b) The center of $G$ contains the center of $K$. So if the former is totally disconnected, then so is the latter.

\section{BIBLIOGRAPHY}

1. C. Chevalley, Theory of Lie groups, I, Princeton, 1946.

2. F. Gantmacher, Canonical representation of automorphisms of a complex semi-simple Lie group, Rec. Math. (Mat. Sbornik) N.S. vol. 5 (1939) pp. 101-144. 85-104.

3. A. M. Gleason, The structure of locally compact groups, Duke Math. J. vol. 18 (1951) pp.

4. - Groups without small subgroups, Ann. of Math. vol. 56 (1952) pp. 193-212.

5. M. Goto, Faithful representations of Lie groups I, Mathematica Japonicae vol. 1 (1948) pp. 107-119.

6. - Linear representations of topological groups, Proc. Amer. Math. Soc. vol. 1 (1950) pp. 425-437.

7. - On the group of automorphisms of a locally compact connected group, Memoirs of the Amer. Math. Soc., no. 14, 1955, pp. 23-29.

8. - Dense imbeddings of locally compact connected groups, Ann. of Math. vol. 61 (1955) pp. 154-169.

9. G. Hochschild, The automorphism group of a Lie group, Trans. Amer. Math. Soc. vol. 72 (1952) pp. 209-216.

10. K. Iwasawa, On some types of topological groups, Ann. of Math. vol. 50 (1949) pp. $507-$ 558.

11. Y. Matsushima, On the decomposition of an (L)-group, J. Math. Soc. Japan vol. 1 (1950) pp. 264-274.

12. D. Montgomery and L. Zippin, Topological transformation groups, New York, 1955.

13. K. Nomizu and M. Goto, On the group of automorphisms of a topological group, Tôhoku Math. J. vol. 2 (1950) pp. 47-50.

14. L. Pontrjagin, Topological groups, Princeton, 1939.

15. A. Weil, L'intégration dans les groupes topologiques et ses applications, Paris, Hermann, 1940.

16. H. Yamabe, On the conjecture of Irwasawa and Gleason, Ann. of Math. vol. 58 (1953) pp. $48-54$. 365

17. - A generalization of a theorem of Gleason, Ann. of Math. vol. 58 (1953) pp. 351-

TUlane UNIVERSITY, New Orleans, La. 